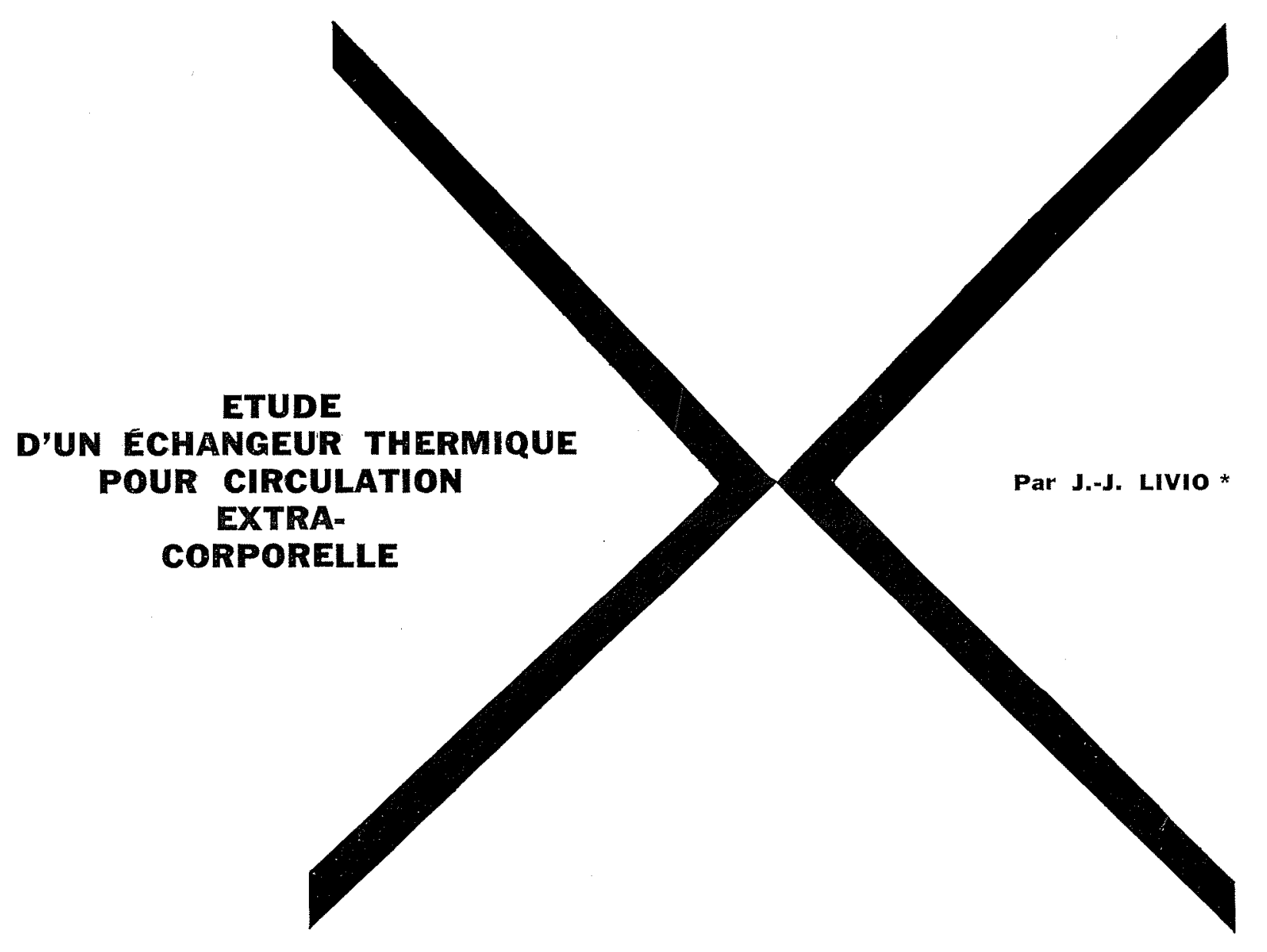

Le probleme du contrôle de la température du patient se pose au cours de toute circulation extracorporelle, soit pour permettre de travailler à un niveau de température différent de la température normale, soit plus simplement pour compenser les pertes caloriques à partir du circuit extra-corporel.

Le moyen d'action le plus commode, tant pour l'efficacité que pour la précision du réglage, est sans doute l'introduction d'un échangeur de chaleur dans le circuit extra-corporel : ceci permet de modifier la température du sang réinjecté au patient au gré des besoins.

Par définition, les échangeurs de chaleur sont des appareils qui permettent de transférer de l'énergie calorifique d'un fluide à un autre, sans qu'il y ait mélange; ils sont caractérisés par :

- un rendement thermique,

- une puissance d'échange thermique,

- une résistance à l'écoulement des fluides,

- un volume de remplissage,

- des conditions particulières à l'usage,

soit un dispositif tel que celui représenté sur la figure 1 .

Un fluide chaud circule dans l'espace extérieur et un fluide froid dans l'espace intérieur. Supposons un régime permanent: la température de chaque point du dispositif est indépendante du temps. Procédons alors à deux coupes transversales délimitant ainsi un segment de très petite longueur; la quantité de chaleur transmise par unité de temps

* Service Universitaire de Chirurgie de Lausanne (Professeur F. SAEGESSER).
(Q) est directement proportionnelle à la surface d'échange (A) et à la différence de température moyenne $(\Delta t)$. Elle peut s'exprimer par l'écfuation suivante :

$$
\mathrm{Q}=\mathrm{K} \cdot \Delta t_{0} \cdot \mathrm{A}
$$

où $\mathrm{K}$ représente le coefficient d'échange global de ce segment. $K$ dépend de la nature des fluides, de leur vitesse, de la nature et de l'épaisseur de la paroi entre autres. Sa valeur peut varier dans le rapport de 1 à 10000 et plus.

En analysant ce coefficient global d'échange, on remarque qu'il dépend de différents coefficients particuliers, soit :

- du coefficient de convection du côté froid,

- du coefficient de conduction de la paroi séparant les deux fluides,

- du coefficient de convection du côté chaud.

La relation qui lie ces coefficients, dans le cas de parois planes ou de tube à parois minces, est exprimée par l'équation suivante :

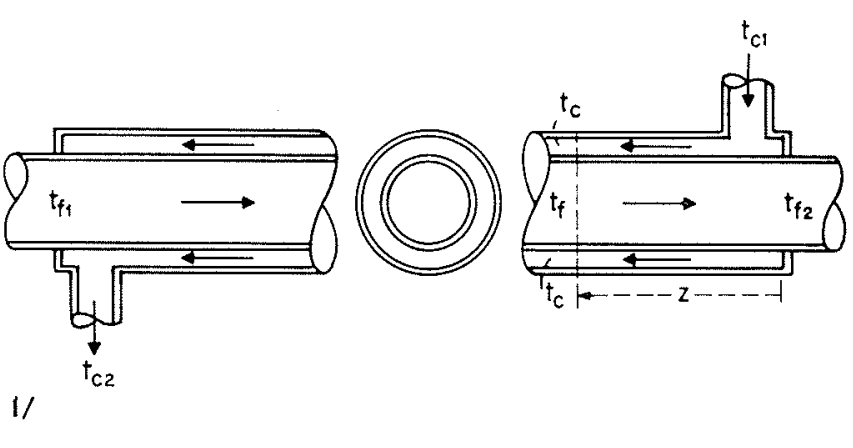


oì

$$
\frac{1}{\mathrm{~K}}=\frac{1}{h_{c}}+\frac{x_{p}}{\lambda_{p}}+\frac{1}{h_{f}}
$$

$h_{i}$ désigne le coefficient de convection du côté chaud;

$h_{f}$ désigne le coefficient de convection du còté froid;

$x_{p}$ désigne l'épaisseur de la paroi;

$\lambda_{p}$ désigne le coefficient de conduction de la paroi.

En résolvant l'écuation par rapport à $K$, on a :

$$
\mathrm{K}=\frac{1}{\left(1 / h_{v}\right)+\left(x_{p} / \lambda_{p}\right)+\left(1 / h_{f}\right)}
$$

K sera done d'autant plus grand que la somme des termes du dénominateur sera petite. La valeur du terme $x_{p} / \lambda_{p}$ est donnée par l'épaisseur et la nature de la paroi.

Les valeurs $h_{c}$ et $h_{j}$ dépendent, entre autres, de :

-_ la viscosité du fluide,

- sa vitesse,

- son coefficient de conduction,

- sa chaleur spécifique,

- l'état de surface de la paroi.

Des formules permettent d'obtenir des valeurs

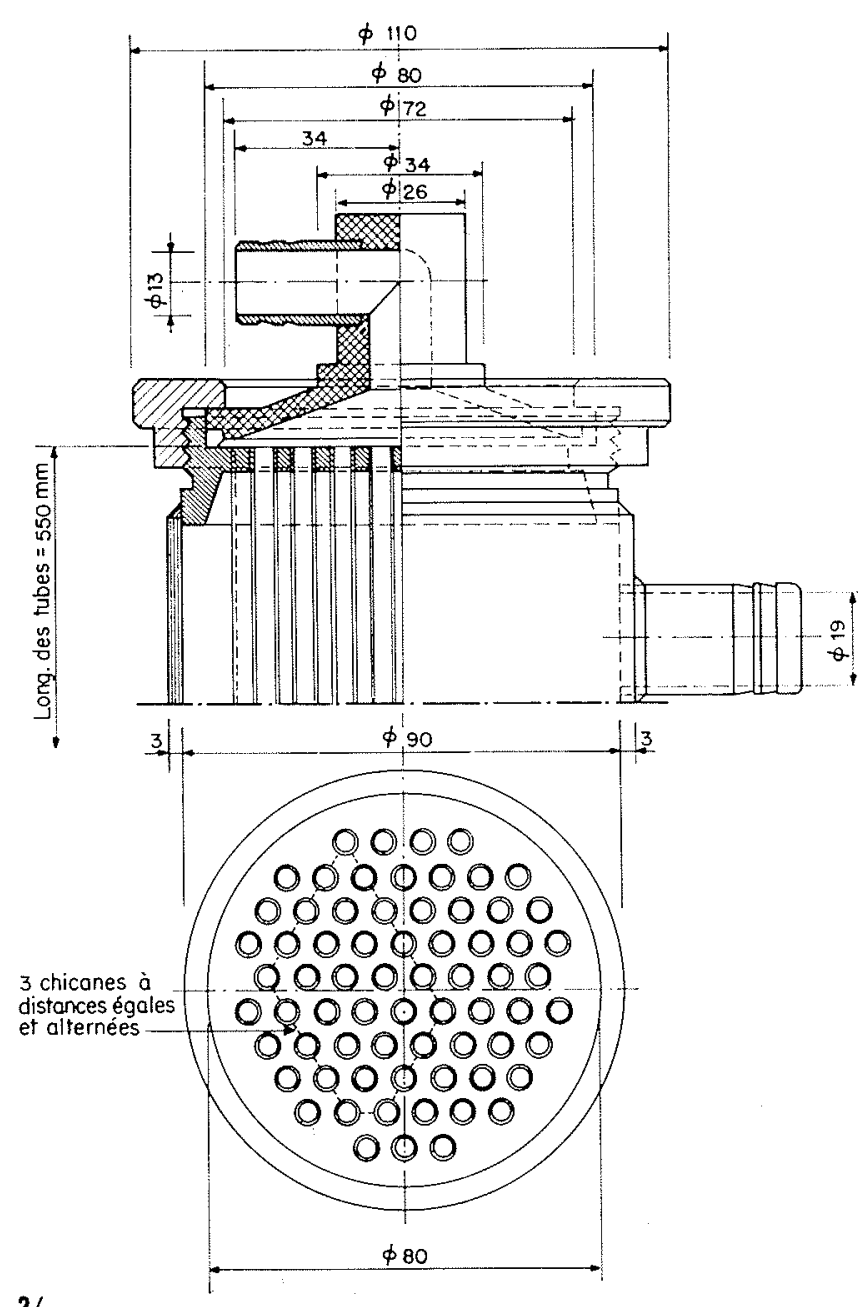

approximatives de $h$ en partant des propriétés du fluide utilisé et de la nature des parois. Nous utilisons celles qui figurent dans l'ouvrage de MacAdams.

Nous avons successivement calculé les valeurs de $h$ dans trois cas supposés de réchauffement pour l'intérieur d'un tube de $10 \mathrm{~mm}$ de diamètre et de $1 \mathrm{~m}$ de long et pour un cas supposé à l'extérieur du tube.

1" du sang à une vitesse de $0,1 \mathrm{~m} / \mathrm{s}$ et à une température initiale de $10^{\circ} \mathrm{C}$;

2" de l'eau dans les mêmes conditions;

$3^{\circ}$ de leau à une vitesse de $1 \mathrm{~m} / \mathrm{s}$ et à une température initiale de $10^{\circ} \mathrm{C}$;

$4^{\circ}$ de l'eau à l'extérieur du tube à une vitesse de $10 \mathrm{~m} / \mathrm{s}$ et à une température initiale de $40^{\circ} \mathrm{C}$.

Les valeurs obtenues ont été les suivantes :

- sang à $0,1 \mathrm{~m} / \mathrm{s}$ :

$$
\mathrm{N}_{\mathrm{R} e}=131 \quad h_{s}=119 \mathrm{cal} / \mathrm{s} \cdot \mathrm{m}^{2} \cdot{ }^{\circ} \mathrm{C}
$$

- eau à $0,1 \mathrm{~m} / \mathrm{s}$ :

$$
\mathrm{N}_{\mathrm{t} e}=904 \quad h_{e l}=117,2 \mathrm{cal} / \mathrm{s} \cdot \mathrm{m}^{2} \cdot{ }^{\circ} \mathrm{C}
$$

-. eau à $1 \mathrm{~m} / \mathrm{s}$ :

$$
\mathrm{N}_{\mathrm{Re}}=9041 \quad h_{c r}=1088 \mathrm{cal} / \mathrm{s} \cdot \mathrm{m}^{2} \cdot{ }^{\circ} \mathrm{C}
$$

- ext. du tube eau à $10 \mathrm{~m} / \mathrm{s}$ :

$$
\mathrm{N}_{\mathrm{R} e}=298000 \quad h_{c e}=9920 \mathrm{cal} / \mathrm{s} . \mathrm{m}^{2} .{ }^{\circ} \mathrm{C}
$$

En caractérisant le tube de séparation des deux fluides, on peut des lors calculer la valeur de $K$. En prenant par exemple pour la paroi un métal relativement mauvais conducteur de la chaleur, comme l'acier inoxydable $\left(\lambda_{p}=3,615 \mathrm{cal} / \mathrm{s} . \mathrm{m} .{ }^{\circ} \mathrm{C}\right)$ avec une épaisseur de $1 \mathrm{~mm}$, on a dans le cas du sang à l'intérieur du tube :

$\mathrm{K}_{s}=\frac{1}{\frac{1}{9920}+\frac{0,001}{3,615}+\frac{1}{119}}=113,9 \mathrm{cal} / \mathrm{s} \cdot \mathrm{m}^{2} \cdot{ }^{\circ} \mathrm{C}$

Supposons maintenant que nous avons réalisé "idéalement» cet échangeur, sans tenir compte des possibilités techniques et technologiques, en prenant une paroi de $0,1 \mathrm{~mm}$ d'épaisseur, faite d'un métal très conducteur comme l'argent :

$$
\left(\lambda=100 \mathrm{cal} / \mathrm{s} . \mathrm{m} .{ }^{\circ} \mathrm{C}\right) .
$$

Avec un etat de surface identique, les valeurs des coefficients $h_{s}$ et $h_{e c}$ restent inchangées; seule la paroi conduit mieux la chaleur. On a :

$$
\begin{gathered}
\mathrm{K}_{s}^{\prime}=\frac{1}{\frac{1}{9920}+\frac{0,0001}{100}+\frac{1}{119}}=117,6 \mathrm{cal} / \mathrm{s} \cdot \mathrm{m}^{2} \cdot{ }^{\circ} \mathrm{C} \\
\mathrm{K}^{\prime}{ }_{s}-\mathrm{K}_{s}=3,2 \mathrm{cal} / \mathrm{s} \cdot \mathrm{m}^{2} \cdot{ }^{\circ} \mathrm{C}
\end{gathered}
$$

ce qui, rapporlé à la moyenne arithmétique :

représenle $3,2 \%$.

$$
\frac{K_{k}+K_{*}^{\prime}}{2}
$$

En bref, on doit dès lors admettre :

$1^{\circ}$ que c'est au droit de la surface de séparation du fluide et de la paroi que se trouve la plus grande résistance à la transmission de la chaleur; 


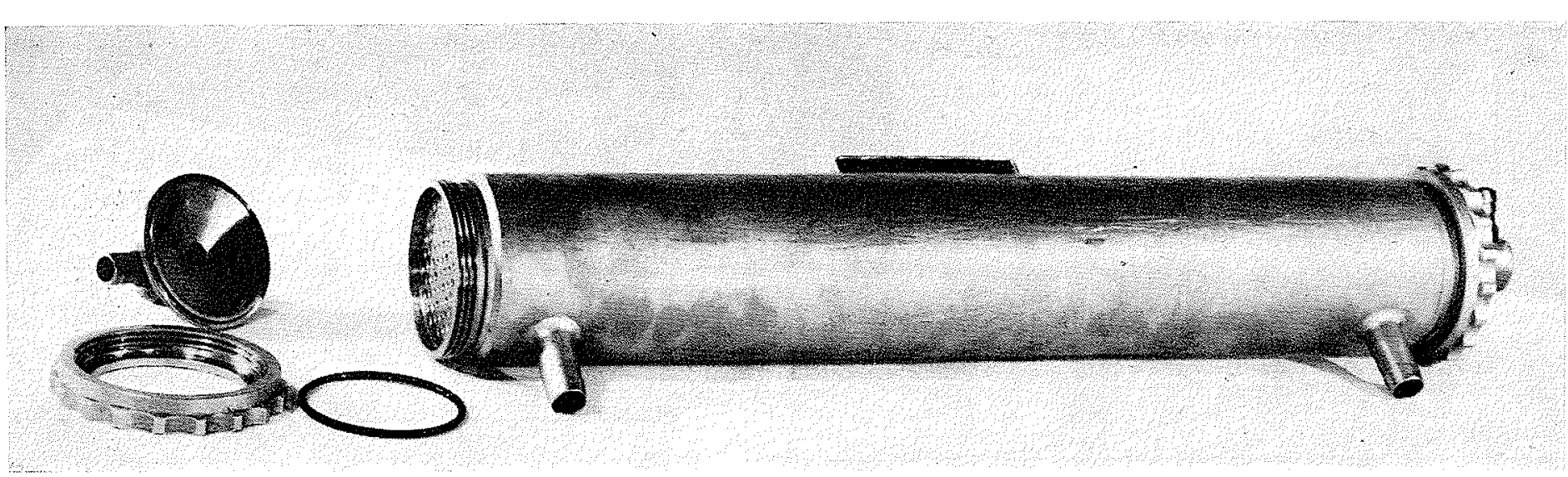

$2^{\circ}$ que la valeur du coefficient de convection en régime laminaire est particulierement faible, principalement parce que les échanges thermiques se font en grande partie par conduction et que les liquides sont de très mauvais conducteurs de la chaleur;

3" que la valeur du coefficient de convection en régime turbulent est notablement plus élevée et que les échanges se font principalement par mélange;

4" que le coefficient de transmission calorifique de la paroi est grandement influencé par le choix du matériau et par l'épaisseur de la paroi, mais que la répercussion sur le coefficient global de l'échangeur est faible dès que l'un des fluides circule en régime laminaire.

Pour ce qui est de la circulation extra-corporelle, un certain nombre de conditions particulières à l'usage nous semblent impératives:

-- il ne doit pas y avoir possibilité de mélange entre le circuit d'eau de conditionnement et le circuit du sang. Il faut donc éviter de séparer ces deux circuils par un système de joints, susceptibles de présenter des fuites par suite d'un montage erroné ou de détérioriation;

- la température des parois en contact avec le sang ne doit pas dépasser $41^{\circ} \mathrm{C}$, ce qui limite la température de l'eau de conditionnement;

-_ les surfaces en contact avec le sang doivent être aussi inertes que possible. L'acier inoxydable de $18 / 8$ ou $18 / 8$ Mo satisfait à cette condition lorsqu'il est poli;

- les vitesses dans le circuit réservé au sang doivent être faibles, de facon à éviter les tourbillons.

D'autres conditions accessoires donneront à l'appareil une aptitude plus ou moins bonne à son utilisation pratique :

- le volume de remplissage doit être minime,

- le nettoyage facile,

-- le montage de l'appareil simple.

La stérilisation à l'autoclave sur un appareil monté est une garantie de stérilité.

Enfin, la résistance à l'écoulement du sang doit être faible si l'appareil est monté sur la ligne vei-

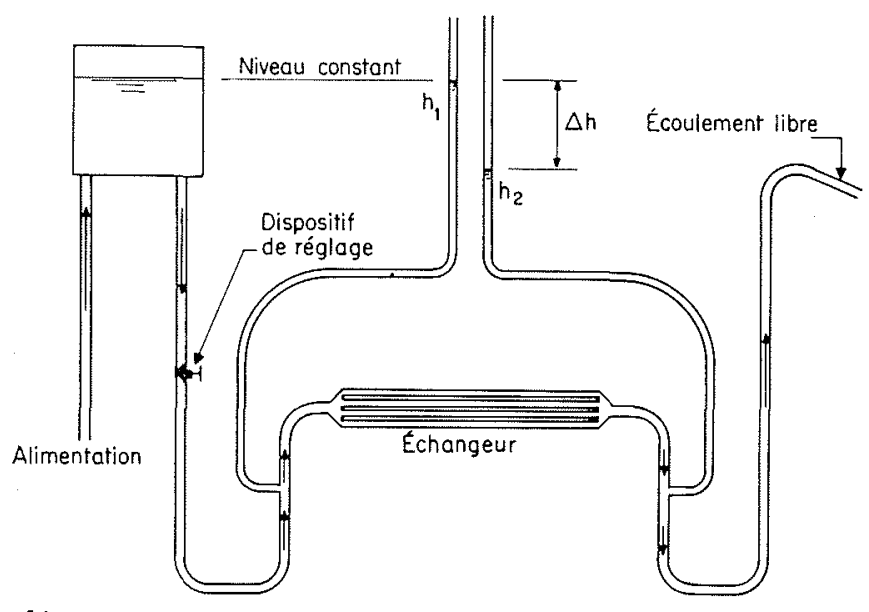

4/

neuse du circuit extra-corporel. La puissance d'échange nécessaire varie suivant l'usage, mais doit permettre de transférer environ 1200 keal en 15 à $25 \mathrm{mn}$.

Pour la réalisation pratique, nous avons choisi un appareil du type multitubulaire qui nous semblait s'accorder le plus facilement aux conditions. Il est entièrement fait en acier inoxydable $18 / 8$ Mo et ne comporte ni brasure, ni soudure à basse température (fig. 2).

Cet appareil est composé d'un faisceau de soixante-neuf tubes, de $3 \mathrm{~mm}$ de diamètre intérieur et de $1 \mathrm{~mm}$ de paroi. Les tubes sont soudés sur deux flasques sous gaz protecteur. Deux fonds de distribution munis d'un coude à $90^{\circ}$ sont maintenus chacun par un écrou. L'étanchéité est réalisée par deux joints toriques. La chambre d'eau est fermée par un tube soudé et comporte trois chicanes. Démonté, l'appareil se compose donc de cinq pièces métalliques et de deux joints toriques (fig. 3 ).

Caractéristiques :

-. volume de remplissage du circuit sanguin. .............. $295 \mathrm{~cm}^{3}$

- surface intérieure des tubes..... $35 \mathrm{dm}^{2}$;

- surface de passage du sang..... 4, 4,84 $\mathrm{cm}^{2}$;

- pression d'essai sur les deux circuits. . . ............ $5 \mathrm{~kg} / \mathrm{cm}^{2}$.

L'essai de l'appareil a porté sur la résistance à l'écoulement d'une part, et la puissance d'échange 

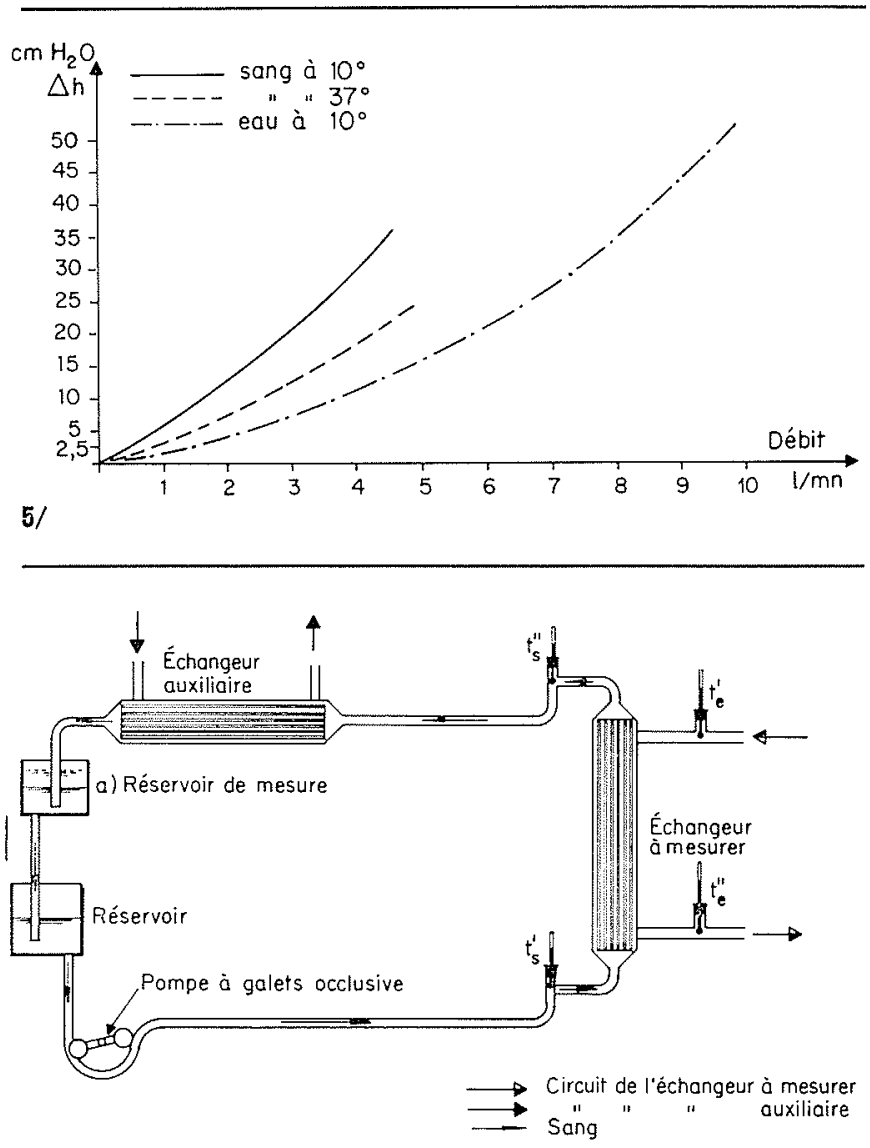

6/

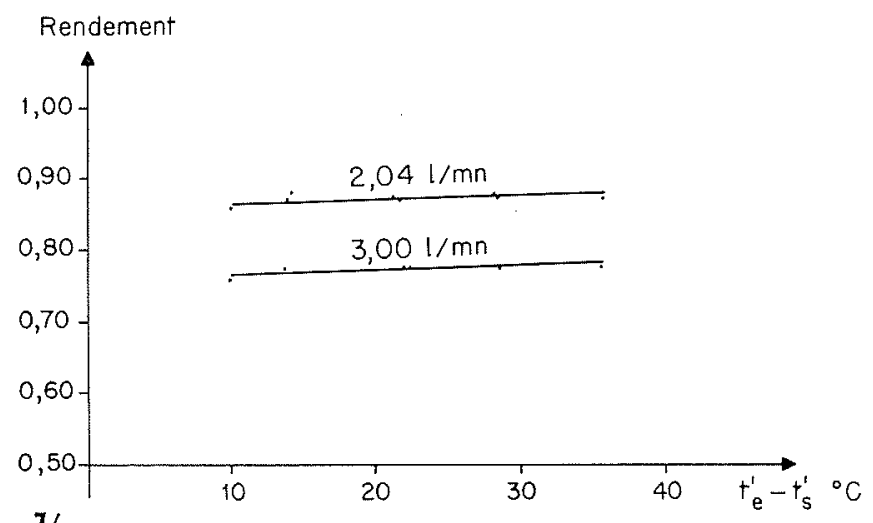

thermique d'autre part, le tout uniquement sur le circuit réservé au sang.

L'essai hydraulique a été conduit avec un dispositif schématiquement représenté sur la figure 4; il a porté sur :

- de l'eau à $10^{\circ} \mathrm{C}$;

- du sang à $10^{\circ} \mathrm{C}$;

- du sang à $37^{\circ} \mathrm{C}$.

Les mesures ont été reportées sur un graphique (fig. 5) où sont notées les pertes de charges en fonction du débit. En analysant les courbes, il est clair que, dans le cas le plus défavorable (sang à $10^{\circ} \mathrm{C}$ ), on obtient des valeurs $\Delta h$ qui se situent à la limite supérieure des normes que nous nous étions fixées.

L'essai calorimétrique a été réalisé à l'aide du dispositif représenté sur la figure 6 ; il a été conduit
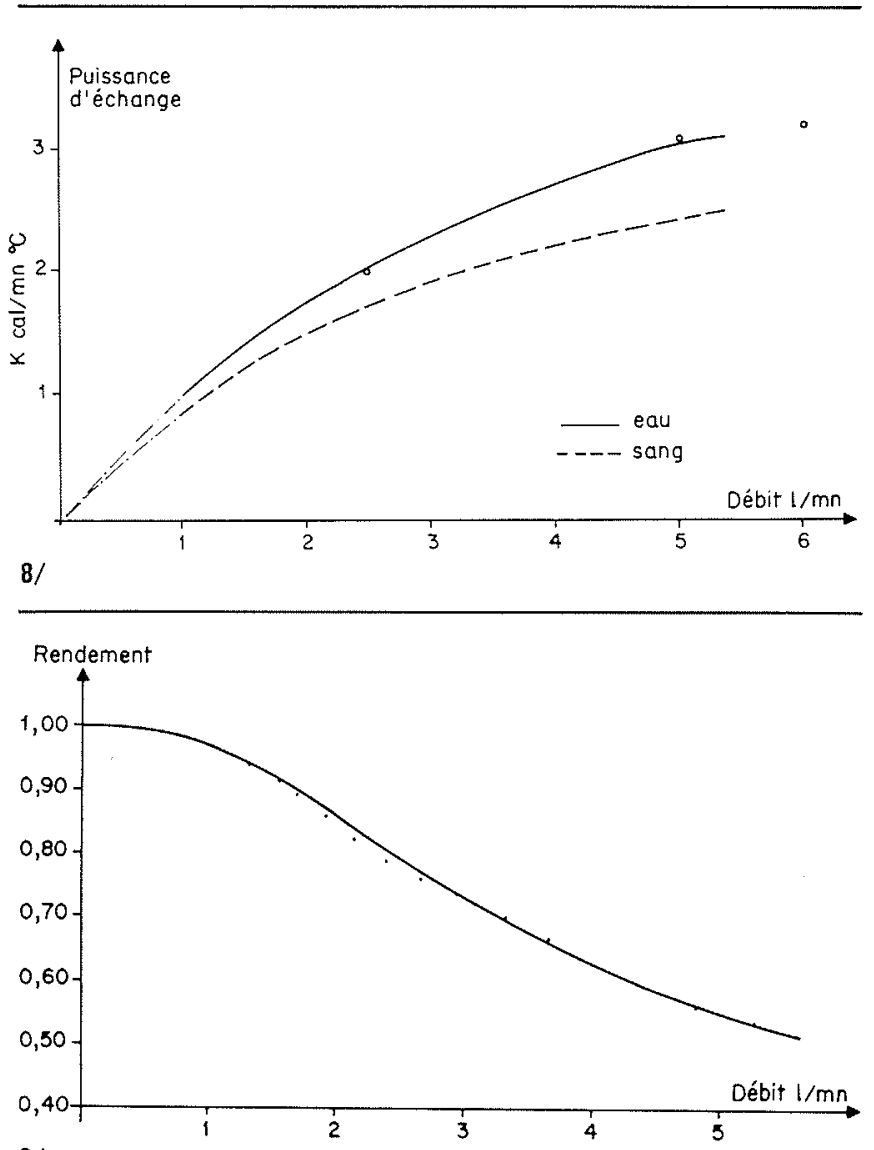

9/

avec du sang et avec de l'eau dans l'espace réservé au sang, pour des débits, dans ce circuit, qui ont varié entre 1 et $5,2 \mathrm{l} / \mathrm{mn}$. Le circuit de conditionnement était alimenté par de l'eau à un débit de $35 \mathrm{l} / \mathrm{mn}$ et à une température initiale de $42^{\circ} \mathrm{C}$.

Les résullats des mesures nous ont permis de calculer la puissance d'échange $P$ d'une part, et le rendement $R$ d'autre part, en fonction du débit en posant :

$$
\mathrm{P}=\frac{c \cdot q\left(t_{s}^{\prime \prime}-t_{s}^{\prime}\right)}{42-t_{s}^{\prime}} \quad \mathrm{R}=\frac{t_{s}^{\prime \prime}-t_{s}^{\prime}}{t_{c}^{\prime}-t_{s}^{\prime}}
$$

A ce propos, la théorie des échangeurs de chaleur montre que, en toute rigueur, $\mathrm{R}$ - donc aussi $\mathrm{P}$ dépend de $t_{e}^{\prime}-t_{s}^{\prime}$. Un essai complémentaire nous a toutefois montré que, dans les conditions envisagées, $P$ et $R$ sont pratiquement indépendants de $t_{e}^{\prime}-t_{s}^{\prime}$ (fig. 7 ).

Nous pouvons donc admettre en première approximation que $P$ et $R$ sont des caractéristiques de l'échangeur pour un débit de sang déterminé, comme c'est la règle dans la littérature médicale.

Sur la figure 8, sont dessinées deux courbes qui représentent la puissance d'échange $P$ en fonction du débit de l'eau et du sang. Sur la courbe de l'eau, les trois points entourés d'un cercle correspondent à une autre méthode de mesure utilisée à titre de contrôle.

La figure 9 montre la courbe de rendement $R$ en fonction du débit; pour du sang, on voit que $R$ est égal ou supérieur à 0,6 jusqu'à un débit de $4,3 \mathrm{l} / \mathrm{mn}$ environ.

L'utilisation clinique a confirmé les données expérimentales : il s'agit en effet d'un échangeur 
puissant, ce que deux exemples suffisent à démontrer :

- P.R. (sténose et insuffisance aortique opérée le 22-7-1963):

- surface corporelle : 2,2 $\mathrm{m}^{2}$;

- refroidissement de 35 à $21^{\circ} \mathrm{C}$ en $6 \mathrm{mn}$;

- réchauffement de 24 à $36^{\circ} \mathrm{C}$ en $15 \mathrm{mn}$.

- A.L. (4 - Fallot opérée le 25-7-1963).
- surface corporelle : $1 \mathrm{~m}^{2}$;

- refroidissement de 36 à $16^{\circ} \mathrm{C}$ en $12 \mathrm{mn}$;

- réchauffement de 12 à $37^{\circ} \mathrm{C}$ en $7 \mathrm{~mm}$.

Le fait que nous utilisons le mème appareil depuis cing ans, sans réparation, permet d'affirmer qu'il est robuste. Enfin, nous ne lui reprochons qu'un seul inconvénient: le nettoyage en est fastidieux.
M. Barres relève que, d'après les auteurs de cette communication, l'efficacité d'un échangeur dépend largement du régime d'ecoulement des fluides et qu'il est fàcheux d'être obligé d'adopter un régime laminaire pour l'écoulement du sang.

Quelles sont les raisons biologiques qui interdisent d'utiliser un régime turbulent? Un tel régime dans l'organisme entraine-t-il des dommages pour le sang?

M. Livio n'est pas certain qu'il existe d'écoulements turbulents dans l'organisme, mais il pense que les éléments figurés sont très sensibles aux tourbillons qui provoquent des distorsions des globules rouges, et que les plaquettes, en particulier, sont très sensibles.

M. VADOT dit être à peu près certain qu'il y a des écoulements turbulents au niveau de l'aorte ascendante ou de la crosse et au niveau des valvules mitrales. Or, il ne semble pas que, dans l'organisme, ce soit catastrophique. Il faut penser que les échanges calorifiques se font au voisinage mème de la paroi et que, même en ayant un régime d'écoulement turbulent, du point de vue des pertes de charge, on reste en régime laminaire dans les couches qui intéressent l'echange themique. On peut se poser la question de savoir si l'on a intérêt à passer du régime laminaire au régime turbulent étanc donné la faible longueur du trajet.

Quant à la disposition à choisir pour un échangeur, il $y$ a tellement de points de vue différents a considérè qu il faut faire un compromis entre des questions de pertes de charge, des questions d'échange thermique et, comme l'a signalé M. Livio, des questions de commodité d'emploi de l'appareil. Le facteur possibilité de nettoyage, de stérilisation de l'appareil n'a pas un caractère physique très noble, mais il faut le considérer.

M. Livio a l'impression qu'en passant à un régime turbulent dans un circuit sanguin, on obtiendrait des rendements bien meilleurs et, donc, un echangeur plus compact, plus petit et nécessitant un volume de remplissage moins gland. Quel est l'avis des hydrauliciens sur ce point?

M. Vavor indique qu'il existe en Amérique des echangeurs de petit volume que l'on n'a pas besoin de démonter, car ils ne servent qu'une fois. Celte solution n'est pas économique car ces échangeurs coûtent $300 \mathrm{~F}$. Avec cet appareil, on arrive à augmenter le nombre de frigories ou de calories que l'on peut échanger dans un volume de sang, car on place, dans un volume faible, wne surface importante avec des epaisseurs de fllms de sanğ extrêmement réduites.

M. Malmejac demande si M. Livio utilise son échangeur de façon préférentielle sur la ligne artérielle ou sur la ligne veineuse suivant les problemes qui se posent.

M. Lrvio répond qu'il l'a utilisé jusquen 1963 uniquement sur la ligne veineuse. A cette époque, on s'est attaqué à la chirurgie valvulaire et on a été gêné par la perte de charge de $35 \mathrm{~cm}$ d'eau avec des débits de 4 à 51 par minute.

Pour cette raison, l'échangeur a été placé dès lors sur la ligne artérielle, où les échanges sont d'ailleurs plus rapides. Il suffit d'avoir un filtre efficace.

M. Chapouthier se demande si le seul paramètre de la lemperature n'est pas insuffisant pour défnir le sang, compte tenu des vitesses de coagulation et de toutes les caractéristiques physiques de ce fluide un peu exceptionnel.
Du sang a $10^{\circ} \mathrm{C}$ est-il te mème chez un individu normal, chez un jeune ou chez un vieillard? Sur quelle base a pu etre determiné le nombre de Reynolds de ces expériences??

M. Livio répond que le sang n'est pas le mème suivant les individus et qu'il a utilise des valeurs moyennes correspondant a des patients bien portants. La viscosité du sang dépend en particulier de la concentration en globules rouges qui est variable d'un patient a l'autre et d'un moment a l'autre chez le meme patient. On a admis, pour le nombre de Resnolds, des valeurs standard de viscosité pour le sang.

M. le Président pense que le problème est compliqué du lait que le sanı́s n'a pas la même température à l'entrée qu’a la sortie et que la viscosité du sang change du simple at double entre 20 et $35^{\circ} \mathrm{C}$. Par conséquent, les conditions d'ecoulement et les conditions de transfert de la chaleur varient tout le long du tube.

M. MaCalre s'exprime en ces mots:

« Je reviens sur me question orale posée ce matin, au sujet des lésions irréversibles gue l'on observe dans le muscle cardiaque après une intervention chirurgicale prolongée.

« Je pense, contrairement à ce qui m'a été fait observer ce matin, que la cause de ces lésions est une question fondamentale, qui mérite d'être posée ici, même s'il est difficile d'y répondre, et mème que cette question est préalable. A quoi servent, en effet, les procédés d'intervention les plus ingénieux, si la nécrose du muscle cardiaque doit être la conséquence d'une intervention chirurgicale?

«Il semble donc qu'il serait intéressant de connaitre les catuses physiologiques de cette nécrose (modification du pH du sang, alimentation en oxygèe, entretien de l'influx nerveux, etc. ou simplement interuption du fonctionnement), mème si le diagnostic des causes n'apporte pas le remède. »

M. le Président indique que, quand le muscle cardiaque ne reçoit pas de sang pendant un temps suffisant, il s'y crée des lésions irréversibles dues à une insuffisance d'apport d'oxygène et de départ des produits du métabolisme local. Par conséquent, même si l'on établissait un temps limite au-delá duquel on craint de voir apparaître des nécroses, il serait sage de rester au-dessous et, ce que les médecins demandent aux ingénieurs, c'es't de leur donnex. la possibilité de réduire ce temps au minimum. Le problème se pose un peu de la mème façon que celui du conditionnement de l'air dans un avion, il faut sarranger pour que, s'il y a une interruption de circulation à faire, la mécanique permette de la taire la plus courte possible.

M. Manion craint que l'échangeur employé par M. Livio soit de trop grande capacité. Pour lhypothermie jusqu'à $10^{\circ} \mathrm{C}$ on n'a pas eu d'accidents particuliers au début; puis, au fur et à mesure de l'expérience, des accidents sérieux sont apparus. On a beaucoup discuté sur leur pathogénie. Il semble bien actuellement admis que le gradient de température du sang et ceux des tissus interviennent dans les accidents qui se produisent en hypothermie profonde et que, précisément, les échangeurs à grande capacité, qu'ils soient à plaques, comme celui de M. VADOT ou à tube, comme celui de M. Lrvio, pourraient être dangereux s'ils étaient employés dans le but d'accélérer la vitesse de refroidissement wu de échauffement. Il faut savoir bien les manier et surtout éviter les gradients trop importants de temperature d'injection du sang et de température des tissus. 


\section{J.-J. LIVIO}

M. Lrvio estime que les accidents dus ì l'hypothermie profonde ont surtout été décelés lors d'arrêts circulatoires totaux, et qu'ils sont moins fréquents et les problèmes des gradients moins importants dès que le sang circule.

Mme Estavone dit avoir observe des accidents lors de clampages relativement courts, oscillant autour de 15 a $20 \mathrm{mn}$, pour des temperatures assez basses, mais avec des gradients de températures assez élevés entre le sang de réinjection et le sang des tissus du cerveau.

M. Du Cailar a travaillé cette question avec le Pr Nègre de Montpelitier. Son expérience, basée sur 150 cas d'hypothermie profonde chez l'homme, lui permet de confirmer les remarques du Professeur Marion. En effet, le risque d'apparition d'accidents nerveux, particulièrement chez les enfants, dont la masse sanguine est minime, oblige à ne refroidir le sang que lentement afin que ne s'installe pas un gradient thermique trop important entre un sang vite refroidi et des tissus plus lents à se refroidir. Un sang trop précocement et trop vite refroidi risque non seulement d'entrainer des phénomènes de vaso-constriction générateurs d'accidents ischémiques au niveau des cellules nerveuses, mais aussi de bloquer les phénomènes de dissociation de l'oxyhémoglobine avant même que les besoins tissulaires ne soient diminués, aggravant de ce fait les lésions induites éventuellement par les phénomènes vaso-moteurs.
Sur une question de M. le Président, M. Du Carlar précise qu'il considère le gradient entre la température du sang et la température des tissus qui peut atteindre 12 a 15 " $\mathrm{C}$ si l'on effectue une hypothermie profonde trop rapide.

M. Mammac indique qu'en ce qui concerne les accidents cérébraux, il faut envisager aussi les éléments figurés. Des numérations de placuettes en cours d'hypothermie ont montré que, lorsqu'on refroidit relativement brutalement le sang, le taux des plaquettes diminue considérablement puisqu'il passe de 300000 à 50000 par $\mathrm{mm}^{3}$ au cours d'une hypothermie prolongée, certaines plaquettes s'agglutinant au niveau du tissu é́rébral. D’autre part, il parât assez vaisemblable que des gradients très importants, au départ d'une hypothermie, puissent entrainer des variations très importantes du système vaso-constricteur et des troubles de la circulation dans certains territoires.

M. le Président remercie les auteurs de cette communication et les personnes ayant pris part à la discussion. Celleci a montré que la question des échangeurs de température est loin d'être réglée et qu'il y aurait lieu de creuser davantage les inconvénients d'un écoulement turbulent du sang, ce rui permettrait de réduire l'encombrement des appareils. Il faudrait aussi savoir si les écarts de température sont fâcheux ou non el quelle est leur amplitude acceptable. 